\title{
Factors affecting the prevention of postpartum hemorrhage in Low- and Middle-Income Countries: A scoping review of the literature
}

\author{
Oliva Bazirete*1, Manassé Nzayirambaho², Marie Chantal Uwimana ${ }^{1}$, Aline Umubyeyi ${ }^{2}$, Evans Marilyn ${ }^{3}$ \\ ${ }^{1}$ Midwifery Department, School of Nursing and Midwifery, College of Medicine and Health Sciences, University of Rwanda, \\ Rwanda \\ ${ }^{2}$ School of Public Health, University of Rwanda, Rwanda \\ ${ }^{3}$ Western University, Canada
}

Received: July 7, 2020

DOI: 10.5430/jnep.v11n1p66
Accepted: September 8, $2020 \quad$ Online Published: October 15, 2020

URL: https://doi.org/10.5430/jnep.v11n1p66

\begin{abstract}
Background and objectives: Postpartum hemorrhage is an important cause of maternal mortality worldwide. A host of literature highlights the difficulty in predicting which women will experience Postpartum hemorrhage. The present study aims at describing the research output on factors affecting the prevention of Postpartum hemorrhage in Low- and Middle- Income Countries.

Methods: A total of 24 published research articles and 2 papers from grey literature published between 2010 and 2019 were retrieved from PubMed, Scopus, CINAHL and Nursing and Allied Health Database (ProQuest). Data were extracted based on main study features and the findings were described narratively. Arksey and O'Malley's framework for scoping studies was used in this review.

Results: Findings from the literature from Low- and Middle- Income Countries are grouped into three themes: Knowledge and understanding about the prevention of Postpartum hemorrhage; Postpartum hemorrhage risk factors among childbearing women; and use of prophylactic uterotonic drugs for PPH prevention and related policies. The majority of studies investigate pharmacological interventions for PPH prevention, while few address the early assessment of risk factors associated with Postpartum hemorrhage for proactive prevention.

Conclusions: The present study suggests that assessing risk factors associated with PPH would be a practical method for early identification of clients at high-risk for developing Postpartum hemorrhage. The present scoping review highlights the paucity of research considering Postpartum hemorrhage risk factors in low- and middle- income countries, particularly in Rwanda. Further qualitative research on the perceptions of health care workers and beneficiaries about Postpartum hemorrhage risk factors is warranted, to enable the development of a tool to assess risk factors associated to Postpartum hemorrhage among childbearing women in Low- and Middle- Income Countries.
\end{abstract}

Key Words: Postpartum hemorrhage, Postpartum complications, Prevention, Low- and Middle- income countries, Scoping

\section{BACKGROUND}

Post-Partum hemorrhage (PPH), commonly defined as "blood loss of $500 \mathrm{ml}$ or more within 24 hours after birth"[1] is a main cause of maternal mortality and morbidity worldwide. ${ }^{[2-5]}$ The amplitude of this condition in the region of sub-Saharan Africa is soaring at $10.5 \% .{ }^{[6]}$ A large retrospec-

\footnotetext{
*Correspondence: Oliva Bazirete; Email: baziretoliva@gmail.com; Address: Midwifery Department, School of Nursing and Midwifery, College of Medicine and Health Sciences, University of Rwanda, Rwanda.
} 
tive cohort study of a maternal mortality audit undertaken in Rwanda found that $70 \%$ of mothers were dying due to direct causes, and $22.7 \%$ of all recorded cases were due to Postpartum hemorrhage. ${ }^{[7]}$ Even though the use of pharmacological interventions to manage the third stage of labour contribute greatly to the prevention of majority of Postpartum hemorrhage, number of women dying from PPH continues to be problematic across the globe. ${ }^{[8]}$ In his paper of introduction to maternal health, Nour ${ }^{[9]}$ highlights that the main reasons for death during childbirth and during the 24 hours following birth, are associated to medical and obstetrical conditions including PPH.

Although some deaths from PPH are unpredictable, Durmaz and $\mathrm{Komurcu}^{[10]}$ note that maternal death due to Postpartum hemorrhage is greatly observed in geographical areas with experiences of difficulties to reach the health facilities and poverty. In addition, inappropriate nutrition, paludism and anaemia may intensify complications of PPH in these under serviced settings. Conversely, maternal mortality from $\mathrm{PPH}$ are rarely observed in environments that have strong socioeconomic environments and satisfactory health services. ${ }^{[11]}$

Vamos, Thompson, Cantor et al. ${ }^{[12]}$ affirm that more than $90 \%$ of hemorrhage-related deaths among childbearing women could be prevented by early assessment of risk factors and timely intervention in clinical care settings. The recognition of factors influencing PPH and its prevention should occur in an ongoing manner throughout the course of antenatal, intrapartum and postpartum care. It has been recommended that midwives and other clinicians take a proactive prevention approach and consider risk factors for PPH during client assessment. ${ }^{[13,14]}$ Evidence suggests that implementing a surveillance system could contribute to identification of the leading sources of morbidity associated with PPH, therefore subsequently assistance could be initiated for improving care, particularly among women who are identified to be at greater risk of Postpartum hemorrhage. ${ }^{[15]}$

The common causes of PPH have been identified globally to be related to uterine atony with or without retained tissues, inadequate blood coagulation, genital tract injuries and traumatism of genital track system. ${ }^{[1,16]}$ Though the occurrence of primary $\mathrm{PPH}$ is not always predictable, the field of PPH prevention is maturing, with improved understanding of PPH risk factors both in developed and in low- and middle- income countries (LMICs). These risk factors could be multifactorial including both demographic and health status factors. However it has been suggested that these risk factors need to be identified and evaluated formally for early prevention of PPH. ${ }^{[17]}$

Previous literature identified PPH risk factors including: Pre- vious history of PPH, pregnancy with more than one fetus, macrosomic fetus, first time mothers, grand multi-parity, advanced age, delivery prior to term, Trauma of the genital tract organs, lack of management of third stage of labor using oxytocics to prevent $\mathrm{PPH}$, induction of labour, caesarean delivery as well as stillbirths. ${ }^{[6,12]}$ There is increasing evidence documenting PPH risk factors in developed countries for early and timely identification of PPH and its prevention. Some LMICs are also embracing different strategies to improve the prevention of PPH and reduce maternal deaths associated with it. It has been noted that, in terms of health care provision and disease prevention, developed countries differ from LMICs. ${ }^{[18]}$ Prata, Bell and Weidert ${ }^{[19]}$ suggest that implementing PPH prevention interventions is essential and needs to be highlighted as an effective way to promote maternal health conditions and reduce maternal mortality. Each country needs to produce its own policies and program compatible to local context to address $\mathrm{PPH}$ in consideration of available resources. A challenging problem which arises in this domain of PPH prevention is a slowed recognition of PPH and lack of timely initiation of treatment, causing deterioration of maternal health condition and death. ${ }^{[20]}$ It is not known how PPH risk factors might be significant especially in LMICs. This gap in knowledge impacts the ability to be proactive in PPH prevention in LMICs. Greater awareness of the evidence about factors associated with $\mathrm{PPH}$ prevention would contribute to more early detection, which implies the need for an overview of the available research evidence in this area. The findings from the present scoping review will therefore enlighten midwifery educational programs and clinical practice particularly in LMICs for an improved proactive PPH prevention.

As reported by Peterson, Pearce, Ferguson et al., ${ }^{[21]}$ the main distinctive aspect of the scoping review studies is to bring a wide view of a broad topic. The present scoping review purpose" $\mathrm{s}$ is to describe the research output on factors affecting PPH prevention in LMICs. It aims at answering the following research questions: "What is already known from the existing research evidence about factors influencing the prevention of PPH in LMICs?" and "Where do the research gaps lie about factors in the prevention of PPH in LMICs?". Levac, Colquhoun and O'Brien ${ }^{[22]}$ report that the commonality of broad research questions is lacking direction, clarity and focus yet needed to enlighten subsequent stages of the study process. The recommendation is to merge the broad research question with a well-defined scope of inquiry. To illuminate the focus of the scoping study and create an efficient search strategy, the authors ${ }^{[22]}$ suggest to define the following three elements: the concept, the population of target, and health outcomes under study. For this review, we 
considered "PPH factors or risk factors" to be the concept under study; "childbearing women in LMICs" as the target population, and "PPH prevention" as the health outcome of interest.

\section{MethodS}

\subsection{Study design}

The present review was undertaken following a methodological process defined by Arksey and O'Malley, ${ }^{[23]}$ discussed by Peterson et al. ${ }^{[21]}$ and taking into considerations the recommendations by Levac et al. ${ }^{[22]}$ To ensure the replicability of our scoping review, a clearly defined study protocol based on the five-steps process for scoping review studies was followed: 1) Identifying the research question(s); 2) Identifying the relevant studies; 3) Study selection; 4) Charting the data; 5) Collating, summarizing, and reporting the data. ${ }^{[23]}$ The use of the sixth step of a 'consultation exercise' to inform and validate findings from the main scoping review is considered as optional according to Arksey and O'Malley. ${ }^{[23]}$ As indicated by literature, ${ }^{[21,24]}$ the main strengths of a scoping study lie in its ability to examine a broader study area to identify research gaps and document on types of evidence that address and enlighten practice in the field under study. Therefore, the present scoping review is designed to describe the research output on factors affecting the prevention of PPH in Low- and Middle- Income Countries.

\subsection{Sources}

To undertake the present scoping review, we initially identified a research question to guide our research process as presented above. We then proceeded to identifying published studies from searches of the following electronic databases: PubMed, Scopus, CINAHL and Nursing and Allied Health Database (ProQuest). Google Scholar was used to locate any other relevant sources within the grey literature. The following key words were used to guide the search: PostPartum hemorrhage ( $\mathrm{PPH})$, prevention, contextual factors, social determinants of health, continuum of care, risk factors, barriers. Sequencing of key word combinations was used. To run the literature search, a combination of two or three words using "AND" was used. As an example "postpartum hemorrhage AND prevention" were combined. To ensure that all relevant data was accessed, the databases were searched multiple times over a period of two months.

\subsection{Study selection and bias assessment}

The study inclusion criteria were: English language articles related to PPH prevention, articles from LMICs, and published between January 2010 till September 2019. The regional scope of our review considered a LMICs' list defined by the World Bank as regions with a "Gross National
Income (GNI) per capita between $\$ 1,026$ and $\$ 3,995$, . ${ }^{25,26]}$ To maximize exploration of updated evidence and other literature pertaining to the research topic, some articles that were found to elaborate at the same time on prevention and on management of PPH were also included but only features associated with PPH prevention were considered for this review. For example, the study conducted by Smith, Currie, Cannon et al. ${ }^{[27]}$ described policies and programs for PPH prevention, management of PPH and pre-eclampsia, but only the sections in relation with PPH prevention were only considered for this scoping review.

The principal investigator (OB: an experienced midwife and educator of midwives in Rwanda) undertook screening of the titles and abstracts of all papers preliminary selected. We retrieved full-text copies of the potentially related articles. We have closely worked with three research supervisors (MN, AU, EM: senior researchers and experts in PPH prevention). The research supervisors helped to resolve uncertainties in the selection process and made spot checks to assess the relevance of the selection. An independent researcher (SM: expert in midwifery) reviewed all full articles selected for inclusion to ensure their relevance to the research questions, and agreed that all the selected articles should be included in the study. The research team met to review the research work on regular basis, and collectively developed a data charting form $^{[22]}$ demonstrating variables to extract and answering the research questions. The following variables were recorded in the form: authors, year of publication, title, study setting, study purpose, study design, data collection, findings, conclusion and factors associated with $\mathrm{PPH}$ prevention.

\subsection{Characteristics of extracted articles}

Using the key search terms through the selected databases, the search process of the present study had identified initially 4415 titles and abstracts. After removing duplicates, 3455 records went forward for initial screening based on titles and abstracts. Of these, 2131 were excluded for being conducted in developed countries, 796 were excluded for not focusing on outcome of interest (PPH prevention). For 528 of these, the full text was evaluated and 315 were excluded for not having the concept of interest (PPH risk factors), 157 were excluded as they were conducted outside the LMIC's, and for nine we could not source the full article. While the remaining 47 went forward for final full text review, 21 were excluded (twelve duplicates and nine non-English). Subsequently, 26 records were included in the review: 24 were published in peer-reviewed journals and 2 were un published reports (i.e. grey literature). A decision flowchart (see Figure 1) outlines the screening process along with selection procedures applied to the records. 


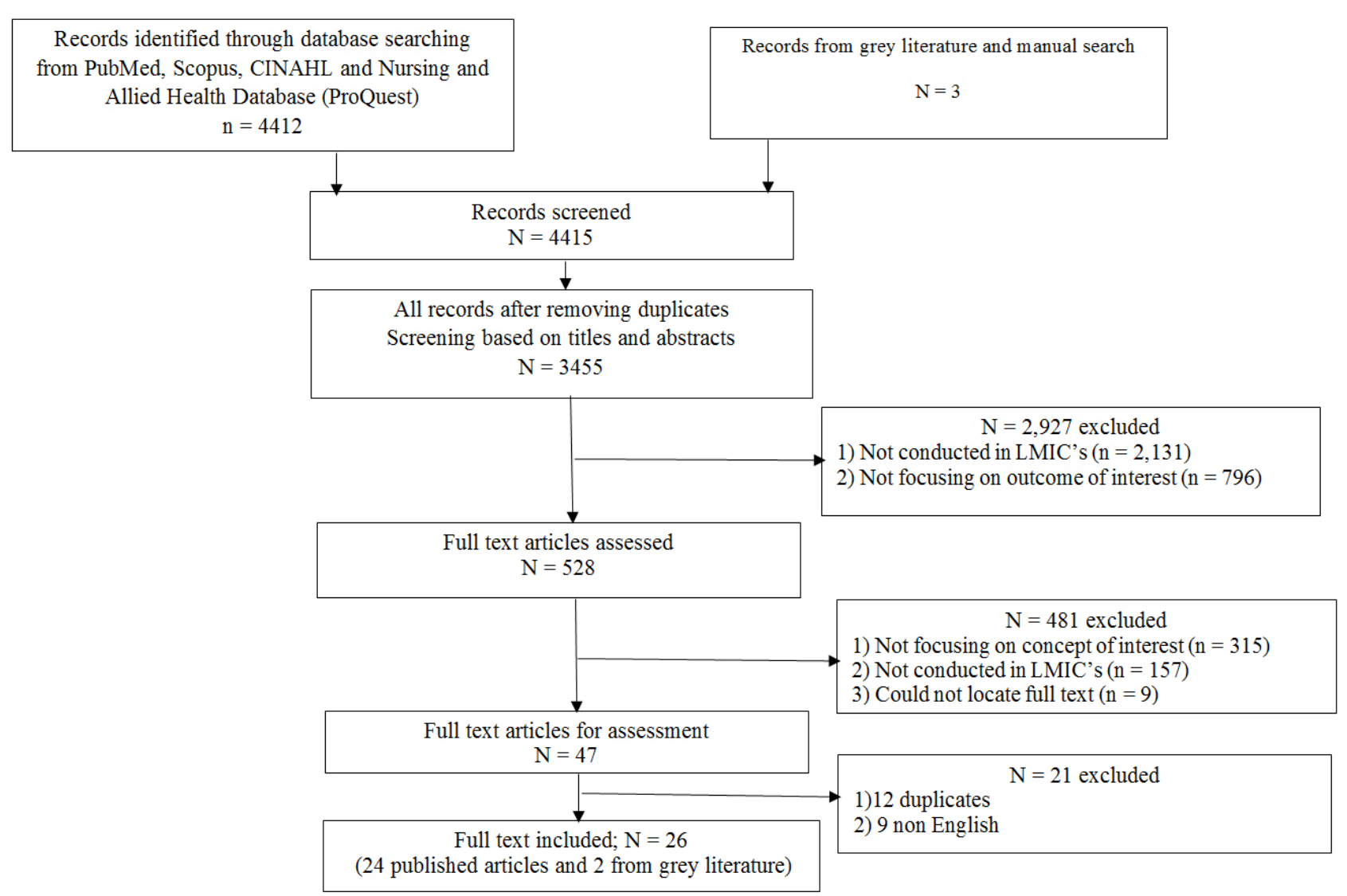

Figure 1. A Flow diagram indicating search process and inclusion

\subsection{Data analysis}

To undertake the data analysis of this review; firstly, characteristics of the study like country, study design, population, information on PPH prevention were extracted and charted. Secondly, we looked at different interventions for PPH prevention and the factors associated with them. In consideration of important attributes of selected studies investigating factors associated with the prevention of PPH, evidence gaps for PPH prevention could be highlighted and a narrative depiction of the current literature was provided. We conducted an inductive content analysis approach to synthesize, analyze and discover the meaning of data extracted, as suggested by Levac et al. ${ }^{[2]}$ To be consistent with inductive content analysis, the entire transcript of extracted data served as a unit of analysis. A spreadsheet was used to summarize the extracted data under specific themes, which were then quantified.

\section{RESULTS}

\subsection{Records identified from grey literature}

The search for unpublished studies revealed two records that inform the factors affecting Postpartum hemorrhage prevention in low- and middle- income countries. The first one is an observational study on immediate postpartum monitoring Published by Sciedu Press conducted in one selected rural district hospital in Rwanda. This project was conducted in 2019 and demonstrated that monitoring of signs associated with postpartum bleeding among women after birth was not frequently done by health care providers. The project recommended to improve the overall monitoring of mothers in postpartum by observing protocols endorsed by the Rwanda Ministry of Health to prevent complication that may occur including PPH. ${ }^{[28]} \mathrm{In}$ addition, we identified a research project that aims to explore barriers to maternal health in Rwanda. ${ }^{[29]}$ The estimated completion date for this study is the end of 2021. This second study involves five districts of the northern province of Rwanda and is largely community-based to investigate barriers encountered by women to prevent maternal near misses including PPH. The preliminary results of this study provided a subgroup data on factors affecting PPH prevention in Rwandan women, and provided imperative insights addressing the present research question.

\subsection{Published studies}

The appendix presents the key features of the 24 published articles. The articles solely focusing on LMIC's represent $91.6 \%$ of all included published studies. Two studies $(8.3 \%)^{[30,31]}$ were large studies combining countries with 
different incomes and their findings also cover LMIC's. These two studies were included in the present review for their added value to factors associated to PPH prevention in LMICs. One paper was a network meta-analysis conducted by Gallos et al. ${ }^{[30]}$ across 53 regions (high-, middle- and low-income countries). A number of 196 trials (including 135,559 women) were conducted to test seven uterotonic agents and placebo or no treatment. The other paper performed a non-inferiority trial to compare the effectiveness of heat-stable carbetocin with those of oxytocin on postpartum hemorrhage after vaginal birth across 10 countries including the LMIC's. Countries involved are Argentina, Egypt, India, Kenya, Nigeria, Singapore, South Africa, Thailand, Uganda, and the United Kingdom. ${ }^{[31]}$

All the 24 articles were published between 2013 and 2019: 12 between 2013 and 2016 and 12 between 2017 and 2019. There was a mix of data collection methods: of the 24 articles, $7(29 \%)$ used quantitative methods, 6 (25\%) were mixed method studies, $4(7 \%)$ were randomized control trials, 4 (17\%) review and analysis of literature, and 3 (12\%) used qualitative methods.

\subsection{Summarizing and reporting findings within themes}

Three main themes emerged from analysis of the extracted data: knowledge and understanding about PPH prevention; risk factors for PPH; and use of prophylactic uterotonic drugs for PPH prevention and related policies. Regarding the focus of extracted records, from the 26 records retrieved including the grey literature, 17 focused on prophylactic use of uterotonic products for the prevention of Postpartum hemorrhage, and 10 were related to knowledge and attitude of health care providers towards PPH prevention. Only 4 articles described risk factors for PPH in LMICs (some articles covered more than one of these themes).

\subsubsection{Knowledge and understanding about PPH preven- tion}

Our findings demonstrate knowledge regarding the PPH prevention among health care providers. It has been reported in a large meta-analysis study on use of uterotonic agents for preventing $\mathrm{PPH},{ }^{[30]}$ that oxytocin is losing its effectiveness if kept at room temperature for a long period or at greater temperatures, which make its usage problematic in low-resource regions. The analysis found evidence that Injectable Oxytocin (10 IU administered intramuscularly or intravenously) stands for the uterotonic of first choice for all births, but entails transport to be stored at temperature between $2{ }^{\circ} \mathrm{C}-8^{\circ} \mathrm{C} .{ }^{[38]} \mathrm{A}$ qualitative inquiry carried out in Ethiopia, India and Myanmar investigated the perspectives and practices of stakeholders towards oxytocin, the requirements for storage and barriers associated, as well as the quality of available product. ${ }^{[50]}$ The study found that in Ethiopia, healthcare providers were aware that it is required that oxytocin should be kept refrigerated, and knew that the product is likely to lose its efficacy once not refrigerated. However, in Myanmar, it was noticed that Oxytocin was stored at room temperature in the labor wards in roughly thirty percent of the health facilities where data collection was conducted. ${ }^{[50]}$ Similarly, in India knowledge of oxytocin storage requirements was shared among the health providers, and was frequently limited to medical doctors and obstetricians/gynecologists. The authors suggest to organize training targeting specific people and produce key advocacy messages emphasizing on the significance of cold chain storage to maintain the quality of oxytocin.

The findings from the present review also demonstrate challenges related to adequate knowledge about PPH prevention among health providers in hospital and community settings. Providers' lack of knowledge of proper indication for uterotonic products used for the prevention of PPH, and their attitudes with regards to the effectiveness of uterotonics products as prophylaxis, were the most shared, described barriers to routine provision of the product in a mixed method study conducted in Sierra Leone. ${ }^{[42]}$ Our findings also show that, in South Ethiopia knowledge and practice of health care providers with regards the prevention of PPH by actively managing the third stage of labor was unsatisfactory. Fewer than half of the health care providers in obstetrics $(37.7 \%)$ had knowledge on how to manage the third stage of labor during childbearing period. ${ }^{[40]}$ To be consistent with knowledge regarding PPH prevention, Smith et al. ${ }^{[32]}$ recommend to set clearly a definition and apply in a similar way the scope of practice in midwifery, in consistence with "International Confederation of Midwives" which recognize essential competencies for the prevention and management of obstetric emergencies.

Our review found that in health communities where competent health care providers are lacking for administration of injectable oxytocin, it is recommended to admister oral Misoprostol (400 $\mu \mathrm{g}-600 \mu \mathrm{g})$ by community health workers or other lay health workers for PPH prevention. ${ }^{[38]}$ To address the challenge of inadequate health literacy in the community, and shortage of skilled health personnel to attend birth, the government of Myanmar trained women who completed secondary education for a period of three months in theory for starting, then they were given three additional months for hands on practical sessions related to care of antenatal period, uncomplicated births and postpartum care. ${ }^{[46]}$ A trusting relationship between auxiliary midwives and midwives has been a catalyst for successful task shifting of administration of Misoprostol by auxiliary midwives in local communities 
of hard to reach geographical area in Myanmar. ${ }^{[46]}$ In contrast to that, a systematic review ${ }^{[45]}$ to investigate gaps and barriers in using misoprostol for the prevention of postpartum hemorrhage in developing countries, fear and confusion were identified amongst people involved in implementation, policy makers and officials at government level. In Tanzania, Webber and Chirangi ${ }^{[47]}$ reported in a pilot study to assess the practicability of self-administration of medications by women to prevent PPH. Government policy in Tanzania prohibits TBAs from administrating Misoprostol in the community, but the study showed that if women are provided with oral Misoprostol during pregnancy, they are definitely both willing and capable to carefully take it at the time of their birth, even when delivery takes place at home, TBA or family member present at the time of delivery.

\subsubsection{Risk factors for PPH among childbearing women}

Findings from this review describe frequent causes of $\mathrm{PPH}$ including atonic uterus, retained tissues, traumatism of genital tract organs and coagulopathy as documented in a study that took place in Uganda to assess incidence and PPH risk factors among women from rural areas ${ }^{[6]}$ and in WHO recommendations on uterotonic use for PPH prevention. ${ }^{[38]}$ In addition to causes of $\mathrm{PPH}$, this review also revealed $\mathrm{PPH}$ risk factors. Vogel et al. ${ }^{[38]}$ reported that most of the women who experience Postpartum hemorrhage don't have identifiable risk factors. ${ }^{[38]}$ But findings from the present scoping review on another hand found studies conducted to assess risk factors and predictors to PPH to improve its prevention. Natakorn et al. ${ }^{[37]}$ in a study to develop a tool to assess the prediction of postpartum bleeding after a vaginal delivery using a retrospective case control study, studied risk factors to PPH identified from literature in Thailand and globally. A multiple logistic regression found eight predictors to blood loss of $300 \mathrm{ml}$ and more after birth. These predictors were: Age of or greater than 35 years, weeks of amenorrhea over 40 , nulliparous women, curettage in previous pregnancy, hypertensive disorders in pregnancy, hemoglobin equal to or less than $10 \mathrm{~g} / \mathrm{dL}$, uterine fundal height of or above 38 centimeters, and having received pethidine for pain management in labor (first stage).

Additional risk factors were identified in a prospective cohort study conducted in Uganda ${ }^{[6]}$ using a calibrated drape placed under-buttocks at childbirth to quantify blood loss of $500 \mathrm{ml}$ or more. After two separate multivariable logistic regression models, other PPH risk factors were found to be clients living with HIV and Caesarean births. Induction of labor and geographical area were also additional risk factors identified after a multivariate logistic regression in a multicountry World Health Organization secondary data analysis of cross-sectional study with all clients giving birth

Published by Sciedu Press and all clients with complicated maternal outcomes. ${ }^{[43]}$ Pre pregnancy Body Mass Index ${ }^{[8]}$ has also been found to be associated with PPH. It was a risk factor of the antepartum period identified in a retrospective cohort study conducted to develop a risk score in relation to women clinical characteristics and past medical history for the prediction of Postpartum hemorrhage in normal labor. Blood loss of $500 \mathrm{ml}$ and more in normal births was measured using a bag. Our findings reveal that the amount of blood loss after birth is believed to be influenced by visual estimation and yet it is the clinical way and most likely the principal approach used for detection of PPH in health facilities. ${ }^{[43]}$

\subsubsection{Use of prophylactic uterotonic drugs for $\mathrm{PPH}$ pre- vention and related policies}

The WHO recommends the use of uterotonic products to prevent $\mathrm{PPH}$ in clients giving birth in health facilities or community settings of the countries from high-income, middleincome or low-income regions. ${ }^{[19,38]}$ As previously demonstrated by our results Oxytocin (10 IU, administered IM or IV) stands as the suggested uterotonic of choice for all deliveries. ${ }^{[19,27,38,42]}$ But due to its heat sensitivity, there have been debates on its cost effectiveness in LMICs. ${ }^{[30,50,52]}$ In these settings, Carbetocin has been found to be as effective as oxytocin without an increase in side effects. ${ }^{[30,31]}$ In settings where oxtytocin is available with existence of cold chain transport and storage available, with health care providers skilled to administer injectable uterotonics, oxtytocin is recommended. ${ }^{[38]}$ In different circumstances, the results from this scoping review show that the heat stable Carbetocin might be more suitable in settings where its cost is affordable, or oral Misoprostol can be used. ${ }^{[38]}$

A meta-analysis by Gallos et al. ${ }^{[30]}$ on uterotonic agents for preventing PPH demonstrated that combinations of regimens are associated with significant side effects. Uterotonic options containing ergometrine may be safely administered if hypertensive diseases can be ruled out before its use. ${ }^{[30,38]}$ Results from this scoping review also demonstrate the possibility and benefits of distribution of misoprostol to clients either for auto-administration, or administration by community health care professionals, auxiliary midwives or community health workers. ${ }^{[33,34,36,38,39,44,47,53,54]}$ A study conducted in Rwanda ${ }^{[34]}$ to investigate the coverage, acceptability, and feasibility of a program for PPH prevention at community and health facility level found that administration of misoprostol by CHWs at the time of delivery for home births achieved reasonable uterotonic coverage. On the other hand, the program achieved satisfactory uterotonic coverage of $82.5 \%$ for all deliveries at the level of health facility due primarily to routine and proper management of the third stage of labor for a high proportion of facility-based deliveries. 
Some challenges have been noted in using prophylactic uterotonics for the prevention of postpartum hemorrhage. Lubinga et al., Rajbhandari et al. and Smith et al. ${ }^{[27,36,44]}$ report insufficient supplies of misoprostol in some settings. Our findings indicate the underuse of available protocols for PPH prevention and the need to review them to enhance their effectiveness and proper use. ${ }^{[42]}$ Prata et al. ${ }^{[19]}$ demonstrated that each country must develop its specific policies in consideration of local context and programs, integrating numerous approaches that pool together the most recent recommendations to address PPH prevention and treatment.

\section{Discussion}

The aim of the present scoping review is to describe the research evidence on factors affecting the prevention of PPH in LMICs. The findings are discussed below in two sections: the first considers risk factors which occur at different stages on the continuum of perinatal care, including the antenatal period, labor and birth, and the postnatal period, while the second section considers different methods for PPH prevention.

\subsection{Risk factors associated with PPH}

It is vital to highlight that recognizing the risk factors and causes of PPH could contribute much to preventing maternal deaths due to PPH. It is demonstrated in our findings that a big number of clients who experience PPH don't demonstrate obvious risk factors. ${ }^{[38]}$ Though the occurrence of PPH is often unpredictable, the literature suggests that mortality can be minimized by identification of the associated risk factors and competent management of the third stage of labor. ${ }^{[55]}$ Merriam, Wright, Siddiq et al. ${ }^{[56]}$ affirm that identifying risk factors during the antenatal period helps to identify women who are at greater risk and suggest the routine use of PPH risk assessment. This review has identified a gap in the routine use of PPH risk assessment in LMICs including Rwanda, and this indicates a need for further investigation.

The potential risk factors identified in the present study include: women's age of 35 years old or above, amenorrhea weeks over 40 weeks, nulliparous women, curettage in previous pregnancy, pregnancy with hypertensive diseases, hemoglobin level less than or equal to $10 \mathrm{~g} / \mathrm{dL}$, uterine fundal height of or greater than 38 centimeters, having received pethidine for pain management during the 1st stage of labor, Caesarean birth; Induction of labor; geographical area; and Body Mass Index before pregnancy. ${ }^{[6,8,37,43]}$ Our results share a number of similarities with previous studies. ${ }^{[13,17,57]}$ In his meta-analysis study, Durmaz ${ }^{[57]}$ addressed the relationship between maternal characteristics (BMI, women age, parity, and hypertensive diseases) and PPH. Maternal age was the only factor not found to have a relationship with PPH. This is in contrast with the findings of Natakorn et al., ${ }^{[37]}$ Sittiparn and Siwadune ${ }^{[8]}$ confirming that advanced women age (greater than 35 years) is a PPH risk factor. Our findings also are in accord with a study conducted in Ethiopia to describe the prevalence and predictors of primary $\mathrm{PPH},{ }^{[58]}$ which suggested that maternal age of or greater than 35 , complicated labor, $\mathrm{PPH}$ in previous pregnancies and deliveries performed with aid of instruments were also predictors of primary PPH. Durmaz and Komurcu ${ }^{[57]}$ recommended that midwives, nurses and obstetricians should cautiously monitor women who present with specific characteristics that are significantly associated with PPH during the antepartum, intrapartum and post-partum period.

Some features of our initial findings align with further studies carried out by Briley et al., ${ }^{[1]}$ Postpartum hemorrhage CPG Work Group and Association of Ontario Midwives. ${ }^{[13]}$ These authors classified PPH risk factors in 3 categories: Pregnancy risk (maternal age, maternal BMI, ethnicity, $\mathrm{PPH}$ in previous pregnancies and assisted conception); Confirmed PPH risk factors acquired during pregnancy (placenta praevia, multiple pregnancy, macrosomic fetus, pre-eclampsia and increased risk of infection) then Confirmed intrapartum PPH risk factors (Fever indicating chorioamnionitis, instrumental deliveries and caesarean section and retained tissues). In contrast to a study by Briley et al. ${ }^{[17]}$ which indicated that induced labor is not complicated with PPH, our findings indicate that induction of labor is likely to be complicated by postpartum bleeding. ${ }^{[43]}$ When comparing our results to those of previous studies, it must be pointed out that duration of labor has been identified to be a PPH risk factor in some other countries. A secondary data analysis of a cohort study including term laboring clients at Washington University Medical Center, showed that PPH risk escalates significantly when the duration of the third stage of labor is equal or greater than 20 minutes. ${ }^{[59]}$ In the same vein, in Norway, Nyfløt, Stray-Pedersen, Forsén et al. ${ }^{[60]}$ found that delayed active phase of labor (duration more than 12 hours) was linked to severe PPH. This factor was not identified in the scope of the reviewed articles of the present scoping review and further investigations could be an interest of research in LMICs.

Similar to previous studies, ${ }^{[1,3,13]}$ findings from the present scoping review classify major causes of PPH into four categories: uterine atony, trauma of the genital tract, retained tissues and maternal coagulopathy. ${ }^{[38]}$ These causes of Postpartum hemorrhage are also in line with the reference manual for training on Basic emergency obstetrics and Neo-natal care adopted by Rwanda Ministry of Health. ${ }^{[61]}$ However considering the major goal of the present study to assess factors 
associated with PPH prevention (antepartum, intrapartum and postpartum periods), these major causes could also be considered among PPH risk factors, as retained placenta was considered to be an intrapartum risk factor. ${ }^{[17]}$

Our findings report also the association between HIV status and PPH. ${ }^{[6]}$ This is comparable to the findings of Curtis, Ayadi, Mkumba et al. ${ }^{[62]}$ study, indicating that HIV is likely to complicate maternal health condition raising up the risk of mortality associated with possible obstetric hemorrhage. But the relationship of HIV status to PPH was not well investigated. This particular finding suggests a need of more research about the underlying mechanism of this association. ${ }^{[62]}$

Numerous factors contribute to the high rates of maternal mortality associated with PPH in LMICs and few studies in these countries have focused on the amplitude and risk factors for PPH. Our review highlights that structural barriers are further complicated by challenges that women may experience like PPH[19]. This finding is complemented by Halle-ekane et al., ${ }^{[5]}$ stressing that a woman's risk of dying after childbirth is not only associated with the amount of blood loss at delivery but also with a woman's general health status, poverty, life style, and nutritional status.

\subsection{Methods for PPH prevention}

Appropriate prevention and treatment of obstetric maternal conditions is important to providing the highest quality of care and avoiding complications. This can only be accomplished if health care providers acquire and keep the necessary competences, to take timely and effective clinical action. ${ }^{[63]}$ Our results demonstrate that knowledge of the heat sensitivity of oxytocin and the potential impacts of inconsistent cold storage on product quality is not widely applied amongst different healthcare professionals. ${ }^{[30,50]}$ This is comparable to findings from Sharifah Nadiah Syed, Nurjasmine Aida and Radiah Abdul ${ }^{[64]}$ in a study to describe the level of knowledge, attitude and practice on PPH among health care professionals in Malaysia where majority of the study participants had an average level of knowledge on prevention of Postpartum hemorrhage and yet possessed a good attitude and practice on its management.

The findings from our scoping review demonstrate that health care providers 'knowledge and practice with regards to active management of the third stage of labor (AMTSL) for PPH prevention is unsatisfactory. ${ }^{[40]}$ This result aligns with previous studies wherein WHO noted gaps in implementing some interventions and accessing the available information among healthcare providers in preventing and managing PPH. ${ }^{[30,65]}$ Moreover, the results from an assessment of quality care at

Published by Sciedu Press national level in Rwanda on the prevention and management of PPH suggest that prevention of PPH using AMTSL needs to be improved. The recommendation was to improve the competences of obstetrical care providers like the correct usage of the AMTSL components which was found to be very low. ${ }^{[66]}$

Previous studies in Rwanda highlighted that ineffective delivery of care at the right time by nurses and midwives and inability to deliver the best package of care to pregnant woman are challenges for the prevention of obstetric complications including PPH. ${ }^{[67,68]}$ When comparing our results to those of previous studies, it must be pointed out that the knowledge of health care providers and their experiences in the field of Postpartum hemorrhage have a great influence in different ways PPH is prevented. This is consistent with Angelina, Kibusi and Mwampagatwa ${ }^{[69]}$ in a study to demonstrate the factors that influence nurses' competencies in PPH prevention and management suggesting that professional qualifications and experience of nurses in maternity units are the main substantial factors influencing their knowledge and skills in implementing different ways of PPH prevention and management.

The most striking results that emerged from this study regarding ways of $\mathrm{PPH}$ prevention is the prophylactic provision of uterotonics immediately after birth. A host of literature, guidelines and policies ${ }^{[38,61,70]}$ indicate that a big number of clients who develop PPH have no previously noticeable risk factors, and as a result the programs for PPH prevention are mostly based on universal use of prophylactic uterotonic drugs for the management of the third stage of labor. New developments in the prevention of PPH and its treatment have been developed in the last decade, and these include advancements done with technology (as an example oxytocin for inhalation and use of the non-pneumatic antishock garment). There have been new strategies for treatment (among others advance distribution of misoprostol for prophylaxis in case of self-administration after delivery, or administered by CHWs, oxytocin administration by usage of Uniiject and care bundles for the management of PPH). ${ }^{[38]}$

Similar to previous studies conducted in $\mathrm{LMICs},{ }^{[13,71,72]}$ our findings highlight that intramuscular or intravenous administration of Oxytocin 10 IU remains the suggested way with regards to using uterotonic products for the prevention of PPH as recommended by WHO. ${ }^{[19,27,38,42]}$ It is suggested that oral administration of Misoprostol can be used for the prevention of Postpartum hemorrhage where oxytocin is lacking or cannot be securely administered. ${ }^{[73]}$ Literature suggests that the main benefit of prophylaxis is the reduction of the rate of anemia due to postpartum. Weeks ${ }^{[74]}$ indicates that 
in cases of higher risk of PPH like placental abruption, low insertion of placenta or placenta praevia, multiple pregnancy or complicated labor) clients are consistently less likely to respond positively to oxytocics, and therefore will require advanced PPH treatments or rescue treatments to avoid increased risks of morbidity and mortality. Therefore, the early identification of PPH risk factors is suggested by the present study so that a particular attention can be paid to cases at high risk of PPH and act on them in a timely way.

\subsection{Strengths and limitations}

To the best of our knowledge, we did not find any study carried out in Rwanda addressing factors associated with PPH prevention. The majority of studies under this review were focusing on the use of prophylactic medication to prevent $\mathrm{PPH}$. It is our belief that the present scoping review is important because it exposes challenges relating to knowledge about PPH and different ways to prevent it. This review suggests that more studies need to be conducted to increase the evidence about the factors associated with PPH in LMICs such as Rwanda. The major limitation to this study is the limited number of publications on early identification of factors associated with PPH prevention in LMICs including the reason to conduct this study.

\subsection{Implication and recommendations}

To prevent and reduce the number and severity of PPH cases, the present study suggests clinical practice and policy implications as suggested by Boyd: ${ }^{[75]}$ the need to change perspective from "reactive" to "proactive" for early detection of clients who are likely to develop PPH. The findings from this study will enhance knowledge of health care providers especially from LMIC's on factors associated with PPH prevention, and therefore inform interventions for the public health with regards to PPH control and prevention. For nurses, midwives and other clinicians, early risk factor identification in the antenatal, intrapartum and postpartum periods may provide an opportunity for timely interventions for PPH prevention. The findings from this study will inform the development and validation of a PPH risk assessment tool, based on PPH risk factors identified to influence the prevention of PPH in the context of LMICs. The tool is expected to improve the quality of information collected about each woman, ensuring better communication among health care providers themselves and with their clients. We propose that qualitative studies involving obstetrical health care professionals in health facilities, community health workers in charge of maternal health, clients who experience PPH and their close relatives could bring new ideas of how early detection of PPH risk factors could contribute to a better prevention of PPH in LMICs such as Rwanda. This study recommends a particular investigation about the association between HIV status and PPH.

\section{Conclusion}

In conclusion, PPH is a major problem in LMICs. Factors associated with its prevention have been identified in this scoping review. This scoping review led to a conclusion confirming that little was known about existing instrument in LMICs that would effectively be used for the assessment of PPH risk factors. Recommendations are made which apply to both governmental and nongovernmental stakeholders to focus on PPH influencing factors for its early detection and prevention.

\section{ACKNOWLEDGEMENTS}

We acknowledge the University of Rwanda and Western University through Training Support Access Model Project for supporting this research. We also acknowledge Ms. Marie Grace Sandra Musabwasoni for her dedication to independently review included articles. Our sincere gratitude goes to Dr. Andrea Nove, technical director at Novametrics in UK for her professional guidance and review of the present paper.

\section{CONFLiCTS OF INTEREST Disclosure}

The authors declare that they have no competing interests.

\section{REFERENCES}

[1] World Health Organization W. WHO recommendations for the pre vention and treatment of postpartum haemorrhage. World Health Organization, 20 Avenue Appia, 1211 Geneva 27, Switzerland: 2012.

[2] World Health Organization W. Time to respond A report on the global implementation of Maternal Death Surveillance and Response. 1211 Geneva 27, Switzerland: WHO Press, World Health Organization, 20169789241511230 .

[3] Say L, Chou D, Gemmill A, et al. Global causes of maternal death: a WHO systematic analysis. The Lancet Global Health. 2014; 2(6): e323-e33. https://doi.org/10.1016/S2214-109X(14) 70227 $-\mathrm{X}$

[4] Bateman BT, Berman MF, Riley LE, et al. The epidemiology of postpartum hemorrhage in a large, nationwide sample of deliveries. Anesthesia and Analgesia. 2010; 110(5): 1368-73. PMid:20237047 https ://doi.org/10.1213/ANE.0b013e3181d74898

[5] Alkema L, Chou D, Hogan D, et al. Global, regional, and national levels and trends in maternal mortality between 1990 and 2015 , with scenario-based projections to 2030: a systematic analysis by the UN Maternal Mortality Estimation Inter-Agency Group. The 
Lancet. 2016; 387(10017): 462-74. https://doi.org/10.1016/ S0140-6736 (15) 00838-7

[6] Ononge S, Mirembe F, Wandabwa J, et al. Incidence and risk factors for postpartum hemorrhage in Uganda. Reproductive Health. 2016; 13: 38. PMid:27080710 https://doi .org/10.1186/s12978-0 16-0154-8

[7] Sayinzoga F, Bijlmakers L, van Dillen J, et al. Maternal death audit in Rwanda 2009-2013: a nationwide facility-based retrospective cohort study. BMJ Open. 2016; 6(1): e009734. PMid:26801466 https://doi.org/10.1136/bmjopen-2015-009734

[8] Sittiparn W, Siwadune T. Risk Score for Prediction of Postpartum Hemorrhages in Normal Labor at Chonburi Hospital. Journal of the Medical Association of Thailand. 2017; 100(4): 382-8.

[9] Nour N. An introduction to maternal mortality. Reviews in obstetrics and gynecology. 2008; 1(2): 77-88.

[10] Durmaz A, Komurcu N. Relationship Between Maternal Characteristics and Postpartum Hemorrhage:A Meta-Analysis Study. Journal of Nursing Research. 2018; 26(5): 362-72. PMid:29219937 https://doi.org/10.1097/jnr.0000000000000245

[11] Hofmeyr J, Qureshi Z. Preventing deaths due to haemorrhage. Best Practice \& Research Clinical Obstetrics \& Gynaecology. 2016; 36 : 68-82. PMid:27450867 https://doi .org/10.1016/j . bpobgyn . 2016.05 .004

[12] Vamos CA, Thompson EL, Cantor A, et al. Contextual factors influencing the implementation of the obstetrics hemorrhage initiative in Florida. Journal of Perinatology: official journal of the California Perinatal Association. 2017; 37(2): 150-6. PMid:27853321 https://doi.org/10.1038/jp. 2016.199

[13] PPH CPG Work Group, Association of Ontario Midwives. Clinical Practice Guideline.Postpartum Hemorrhage. 2016.

[14] Mavrides E, Allard S, Chandraharan E, et al. Prevention and Management of Postpartum Haemorrhage: Green-top Guideline No. 52. BJOG: an international journal of obstetrics and gynaecology. 2016. p. e106-e49.

[15] Rocha Filho EA, Costa ML, Cecatti JG, et al. Severe maternal morbidity and near miss due to postpartum hemorrhage in a national multicenter surveillance study. International Journal of Gynaecology \& Obstetrics. 2015; 128(2): 131-6. PMid:25468058 https://doi.org/10.1016/j.ijgo.2014.08.023

[16] Weeks A. Women's rights are integral to prevention and treatment of PPH. BJOG: an international journal of obstetrics and gynaecology. 2015; 122(2): 212. PMid:25546045 https ://doi.org/10.1111/ 1471-0528.13227

[17] Briley A, Seed PT, Tydeman G, et al. Reporting errors, incidence and risk factors for postpartum haemorrhage and progression to severe PPH: a prospective observational study. BJOG: an international journal of obstetrics and gynaecology. 2014; 121(7): 876-88. PMid:24517180 https://doi.org/10.1111/1471-0528.1258 8

[18] Calvert C, Thomas SL, Ronsmans C, et al. Identifying Regional Variation in the Prevalence of Postpartum Haemorrhage: A Systematic Review and Meta-Analysis. PLoS One. 2012; 7(7): 1-10. PMid:22844432 https://doi.org/10.1371/journal.pone.0 041114

[19] Prata N, Bell S, Weidert K. Prevention of postpartum hemorrhage in low-resource settings: current perspectives. Int J Womens Health. 2013; 5: 737-52. PMid:24259988 https://doi.org/10.2147/ I JWH. S51661

[20] Andrikopoulou M, D'Alton ME. Postpartum hemorrhage: early identification challenges. Seminars in Perinatology. 2019; 43(1): 11-7. PMid:30503400 https://doi.org/10.1053/j. semperi. 2018 .11 .003

Published by Sciedu Press
[21] Peterson J, Pearce PF, Ferguson LA, et al. Understanding scoping reviews: Definition, purpose, and process. J Am Assoc Nurse Pract. 2017; 29(1): 12-6. PMid:27245885 https://doi.org/10.1002/ $2327-6924.12380$

[22] Levac D, Colquhoun H, O'Brien KK. Scoping studies: advancing the methodology. Implementation Science. 2010; 5(69): 1-9. PMid:20854677 https://doi .org/10.1186/1748-5908-5-69

[23] Arksey H, O'Malley L. SCOPING STUDIES: TOWARDS A METHODOLOGICAL FRAMEWORK. International Journal of Social Research Methodology: Theory \& Practice. 2005; 8(1): 19-32. https://doi.org/10.1080/1364557032000119616

[24] The Joanna Briggs Institute J. Joanna Briggs Institute Reviewers' Manual: 2015 edition/supplement. Methodology for JBI Scoping Reviews. 2015.

[25] World Bank W. New country classifications by income level: 2018-2019: World Bank data team; 2018. Available from: https://blogs.worldbank.org/opendata/new-country-classificationsincome-level-2018-2019.

[26] World Bank W. Classifying countries by income: World Bank; 2019 [updated September 2019; cited 20207 September 2020]. Available from: https://datatopics.worldbank.org/world-develop ment-indicators/stories

[27] Smith JM, Currie S, Cannon T, et al. Are national policies and programs for prevention and management of postpartum hemorrhage and preeclampsia adequate? A key informant survey in 37 countries. Glob Health Sci Pract. 2014; 2(3): 275-84. PMid:25276587 https://doi.org/10.9745/GHSP-D-14-00034

[28] Manayacu B, Mukamazimpaka V, Uwayezu A, et al. Observational Study on the Immediate Postpartum Monitoring at Kabutare District Hospital, Rwanda. 2019.

[29] Bagambe Gatsinzi P, Umubyeyi A, Luginaah I. Exploration of barriers to maternal health in Rwanda. [PHD Thesis]. In press 2019.

[30] Gallos ID, Papadopoulou A, Man R, et al. Uterotonic agents for preventing postpartum haemorrhage: a network meta-analysis. The Cochrane Database of Systematic Reviews. 2018; 12: CD011689.

[31] Widmer M, Piaggio G, Nguyen TMH, et al. Heat-Stable Carbetocin versus Oxytocin to Prevent Hemorrhage after Vaginal Birth. The New England Journal of Medicine. 2018; 379(8): 743-52. PMid:29949473 https://doi.org/10.1056/NEJMoa1805489

[32] Smith JM, Dimiti A, Dwivedi V, et al. Advance distribution of misoprostol for the prevention of postpartum hemorrhage in South Sudan. International Journal of Gynaecology and Obstetrics: the official organ of the International Federation of Gynaecology and Obstetrics. 2014; 127(2): 183-8. PMid:25051905 https://doi.org/10.101 6/j.ijgo.2014.05.016

[33] Priya GP, Veena P, Chaturvedula L, et al. A randomized controlled trial of sublingual misoprostol and intramuscular oxytocin for prevention of postpartum hemorrhage. Archives of Gynecology and Obstetrics. 2015; 292(6): 1231-7. PMid:25990482 https://doi. org/10.1007/s00404-015-3763-5

[34] Dao B, Ngabo F, Zoungrana J, et al. Increasing Access to Prevention of Postpartum Hemorrhage Interventions for Births in Health Facilities and at Home in Four Districts of Rwanda. African Journal of Reproductive Health December. 2015; 19(4): 58-67.

[35] Elbohoty AE, Mohammed WE, Sweed M, et al. Randomized controlled trial comparing carbetocin, misoprostol, and oxytocin for the prevention of postpartum hemorrhage following an elective cesarean delivery. International Journal of Gynaecology and Obstetrics: the official organ of the International Federation of Gynaecology and Obstetrics. 2016; 134(3): 324-8. PMid:27350226 https://doi.org/10.1016/j.ijgo.2016.01.025 
[36] Lubinga SJ, Atukunda EC, Wasswa-Ssalongo G, et al. Potential CostEffectiveness of Prenatal Distribution of Misoprostol for Prevention of Postpartum Hemorrhage in Uganda. PloS one. 2015; 10(11): e0142550. PMid:26560140 https://doi.org/10.1371/journa 1.pone. 0142550

[37] Natakorn IT, Ratanasiri A, Nutravong T, et al. Risk Scoring System for the Prediction of Postpartum Blood Loss over $300 \mathrm{~mL}$ at Chiang Rai Regional Hospital. Siriraj Medical Journal. 2019; 71(1): 110-6. https ://doi.org/10.33192/Smj . 2019.17

[38] Vogel JP, Williams M, Gallos I, et al. WHO recommendations on uterotonics for postpartum haemorrhage prevention: what works, and which one? BMJ Global Health. 2019; 4(2): e001466. PMid:31139461 https://doi.org/10.1136/bmjgh-2019-001 466

[39] Hobday K, Hulme J, Homer C, et al. "My job is to get pregnant women to the hospital": a qualitative study of the role of traditional birth attendants in the distribution of misoprostol to prevent post-partum haemorrhage in two provinces in Mozambique. Reproductive Health. 2018; 15(1): 174. PMid:30326927 https : //doi.org/10.1186/s12978-018-0622-4

[40] Tenaw Z, Yohannes Z, Amano A. Obstetric care providers' knowledge, practice and associated factors towards active management of third stage of labor in Sidama Zone, South Ethiopia. BMC Pregnancy and Childbirth. 2017; 17(1): 292. PMid:28882109 https: //doi.org/10.1186/s12884-017-1480-8

[41] Charles D, Anger H, Dabash R, et al. Intramuscular injection, intravenous infusion, and intravenous bolus of oxytocin in the third stage of labor for prevention of postpartum hemorrhage: a threearm randomized control trial. BMC Pregnancy and Childbirth. 2019; 19(1): 38. PMid:30658605 https : //doi.org/10.1186/s12884 $-019-2181-2$

[42] Natarajan A, Ahn R, Nelson BD, et al. Use of prophylactic uterotonics during the third stage of labor: a survey of provider practices in community health facilities in Sierra Leone. BMC Pregnancy and Childbirth. 2016; 16: 23. PMid:26821645 https://doi.org/10 .1186/s12884-016-0809-z

[43] Sheldon WR, Blum J, Vogel JP, et al. Postpartum haemorrhage management, risks, and maternal outcomes: findings from the World Health Organization Multicountry Survey on Maternal and Newborn Health. BJOG: an international journal of obstetrics and gynaecology. 2014; 121 Suppl 1: 5-13. PMid:24641530 https: //doi.org/10.1111/1471-0528.12636

[44] Rajbhandari SP, Aryal K, Sheldon WR, et al. Postpartum hemorrhage prevention in Nepal: a program assessment. BMC Pregnancy and Childbirth. 2017; 17(1): 169. PMid:28583092 https: //doi.org/10.1186/s12884-017-1347-z

[45] Samnani A, Rizvi N, Ali TS, et al. Barriers or gaps in implementation of misoprostol use for post-abortion care and post-partum hemorrhage prevention in developing countries: a systematic review. Reproductive Health. 2017; 14(1): 139. PMid:29078777 https ://doi.org/10.1186/s12978-017-0383-5

[46] Than KK, Mohamed Y, Oliver V, et al. Prevention of postpartum haemorrhage by community-based auxiliary midwives in hard-toreach areas of Myanmar: a qualitative inquiry into acceptability and feasibility of task shifting. BMC Pregnancy and Childbirth. 2017; 17(1): 146. PMid:28514959 https ://doi.org/10.1186/s12884 $-017-1324-6$

[47] Webber GC, Chirangi B. Women's health in women's hands: a pilot study assessing the feasibility of providing women with medications to reduce postpartum hemorrhage and sepsis in rural Tanzania. Health Care for Women International. 2014; 35(7-9): 758-
70. PMid:24786175 https : //doi .org/10.1080/07399332. 201 4.915843

[48] Webber G, Chirangi B. "Please Do Not Forget Us" - Views of Women, Nurses, and Traditional Birth Attendants on Community Distribution of Medications to Prevent Postpartum Hemorrhage and Sepsis: A Qualitative Pilot Study in Rural Tanzania. Journal of Women's Health Care. $2014 ; 3(4)$ : 1-7.

[49] Ndirangu G, Gichangi A, Kanyuuru L, et al. Using Young Mothers' Clubs to Improve Knowledge of Postpartum Hemorrhage and Family Planning in Informal Settlements in Nairobi, Kenya. Journal of Community Health. 2015; 40(4): 692-8. PMid:25585809 https://doi.org/10.1007/s10900-014-9986-8

[50] Oliver VL, Lambert PA, Than KK, et al. Knowledge, perception and practice towards oxytocin stability and quality: A qualitative study of stakeholders in three resource-limited countries. PloS one. 2018; 13(9): e0203810. PMid:30252860 https://doi.org/10.1371/ journal.pone. 0203810

[51] Kapungu CT, Mensah-Homiah J, Akosah E, et al. A communitybased continuum of care model for the prevention of postpartum hemorrhage in rural Ghana. International Journal of Gynaecology and O-bstetrics: the official organ of the International Federation of Gynaecology and Obstetrics. 2013; 120(2): 156-9. PMid:23199804 https://doi.org/10.1016/j.ijgo.2012.08.021

[52] Prata N, Passano P, Bell S, et al. New hope: community-based misoprostol use to prevent postpartum haemorrhage. Health Policy and Planning. 2013; 28(4): 339-46. PMid:22879523 https : //doi.org/10.1093/heapol/czs068

[53] Rwanda Ministry of Health R. KWITA KU MUGORE UTWITE, UWABYAYE N'URUHINJA MU RUGO. 2015.

[54] Rwanda Ministry of Health R. Maternal Newborn and Child Health Strategic Plan (2018-2024). 2018.

[55] Halle-Ekane G, Emade F, Bechem N, et al. Prevalence and Risk Factors of Primary Postpartum Hemorrhage after Vaginal Deliveries in the Bonassama District Hospital, Cameroon. International Journal of TROPICAL DISEASE \& Health. 2016; 13(2): 1-12. https : //doi .org/10.9734/IJTDH/2016/23078

[56] Merriam AA, Wright JD, Siddiq Z, et al. Risk for postpartum hemorrhage, transfusion, and hemorrhage-related morbidity at low, moderate, and high volume hospitals. The journal of maternal-fetal \& neonatal medicine: the official journal of the European Association of Perinatal Medicine, the Federation of Asia and Oceania Perinatal Societies, the International Society of Perinatal Obstet. 2018; 31(8): 1025-34. PMid:28367647 https : //doi.org/10.1080/14 767058.2017 .1306050

[57] Durmaz A, Komurcu N. Relationship Between Maternal Characteristics and Postpartum Hemorrhage: A Meta-Analysis Study. The Journal of Nursing Research. 2018; 00(0): 1-11.

[58] Kebede BA, Abdo RA, Anshebo AA, et al. Prevalence and predictors of primary postpartum hemorrhage: An implication for designing effective intervention at selected hospitals, Southern Ethiopia. PloS one. 2019; 14(10): e0224579. PMid:31671143 https : //doi.org/ 10.1371/journal pone. 0224579

[59] Frolova AI, Stout MJ, Tuuli MG, et al. Duration of the Third Stage of Labor and Risk of Postpartum Hemorrhage. Obstetrics and Gynecology. 2016; 127(5): 951-6. PMid:27054942 https: //doi.org/10.1097/A0G.0000000000001399

[60] Nyfløt LT, Stray-Pedersen B, Forsén L, et al. Duration of labor and the risk of severe postpartum hemorrhage: A case-control study. PloS one. 2017; 12(4): 1-10. PMid:28384337 https ://doi.org/10.1 371/journal.pone. 0175306 
[61] Rwanda Ministry of Health R. Formation Continue en Soins Obstétricaux et Néonatals d'Urgence de Base. In: Maternal NBCaCHD, editor. Kigali, Rwanda. 2016. p. 1-145.

[62] Curtis M, Ayadi AE, Mkumba G, et al. Association between severe obstetric hemorrhage and HIV status. In: Obstetrics IFoGa, editor. 2014.

[63] Vadnais MA, Dodge LE, Awtrey CS, et al. Assessment of long-term knowledge retention following single-day simulation training for uncommon but critical obstetrical events. The Journal of MaternalFetal \& Neonatal Medicine. 2012; 25(9): 1640-5. PMid:22191668 https://doi.org/10.3109/14767058.2011.648971

[64] Sharifah Nadiah Syed I, Nurjasmine Aida J, Radiah Abdul G. Knowledge, Attitude and Practice on Postpartum Haemorrhage among Women in Kuantan, Pahang, Malaysia. International Journal of Research in Pharmaceutical Sciences. 2019; 10(3): 2329-33. https: //doi.org/10.26452/ijrps.v10i3.1474

[65] Tuncalp, Were WM, MacLennan C, et al. Quality of care for pregnant women and newborns-the WHO vision. BJOG: an international journal of obstetrics and gynaecology. 2015; 122(8): 1045-9. PMid:25929823 https://doi.org/10.1111/1471-0528.1345 1

[66] Favero R, Ngabo F, Zoungrana J, et al. O761 POSTPARTUM HEMORRHAGE PREVENTION AND MANAGEMENT REQUIRE STRENGTHENING: FINDINGS FROM A NATIONAL QUALITY OF CARE ASSESSMENT IN RWANDA. International Journal of Gynecology \& Obstetrics. 2012; 119: S529-S. https ://doi.or $\mathrm{g} / 10.1016 / \mathrm{S} 0020-7292(12) 61191-6$

[67] Sengoma JPS, Krantz G, Nzayirambaho M, et al. Prevalence of pregnancy-related complications and course of labour of surviving women who gave birth in selected health facilities in Rwanda: a health facility-based, cross-sectional study. BMJ Open. 2017; 7: 119. PMid:28694344 https://doi.org/10.1136/bmjopen-201 6-015015
[68] Rulisa S, Umuziranenge I, Small M, et al. Maternal near miss and mortality in a tertiary care hospital in Rwanda. BMC Pregnancy and Childbirth. 2015; 15: 203. PMid:26334634 https: //doi.org/10.1186/s12884-015-0619-8

[69] Angelina JA, Kibusi SM, Mwampagatwa I. Factors influencing nurses' knowledge and skills in the prevention and management of postpartum haemorrhage. African Journal of Midwifery and Women's Health. 2019; 13(4). https : //doi.org/10.12968/ajm w. 2018.0018

[70] RANZCOG Board and Council. R. Management of Management Postpartum Haemorrhage (PPH). In: Gynecologists TRAaNZCoOa, editor. New Zeland. 2017.

[71] Bartlett L, Cantor D, Lynam P, et al. Facility-based active management of the third stage of labour: assessment of quality in six countries in sub-Saharan Africa. Bulletin of the World Health Organization. 2015; 93(11): 759-67. PMid:26549903 https : //doi .org/10 $.2471 /$ BLT. 14.142604

[72] Westhoff G, Cotter AM, Tolosa JE. Prophylactic oxytocin for the third stage of labour to prevent postpartum haemorrhage. The Cochrane Database of Systematic Reviews. 2013(10): CD001808. https://doi.org/10.1002/14651858.CD001808.pub2

[73] Lalonde A, International Federation of G, Obstetrics. Prevention and treatment of postpartum hemorrhage in low-resource settings. International journal of gynaecology and obstetrics: the official organ of the International Federation of Gynaecology and Obstetrics. 2012; 117(2): 108-18. PMid:22502595 https://doi .org/10.1016/j. ijgo.2012.03.001

[74] Weeks A. The prevention and treatment of postpartum haemorrhage: what do we know, and where do we go to next? BJOG: an international journal of obstetrics and gynaecology. 2015; 122: 202-12. PMid:25289730 https://doi.org/10.1111/1471-0528.1309 8

[75] Boyd J. Introducing a Structured Checklist in the Consultant Led Maternity Unit. 2015. 